\title{
The Effects and Measures of Auricular Acupressure and Interactive Multimedia for Smoking Cessation in College Students
}

\author{
Mei-Ling Yeh,, Pei-Lan Wang, ${ }^{2}$ Jaung-Geng Lin, ${ }^{3,4}$ and Mei-Ling Chung ${ }^{1}$ \\ ${ }^{1}$ School of Nursing, National Taipei University of Nursing and Health Sciences, 365, Ming-Te Road, Pei-Tou, Taipei 112, Taiwan \\ ${ }^{2}$ Department of Nursing, Hsin Sheng College of Medical Care and Management, Taoyuan, Taiwan \\ ${ }^{3}$ Graduate Institute of Acupuncture Science, China Medical University \& China Medical University Hospital, Taichung, Taiwan \\ ${ }^{4}$ School of Chinese Medicine and Acupuncture Science, China Medical University, 91 Hsueh-Shih Road, Taichung 40402, Taiwan
}

Correspondence should be addressed to Jaung-Geng Lin; jglin@mail.cmu.edu.tw and Mei-Ling Chung; mlchuang@ntunhs.edu.tw Received 28 March 2014; Accepted 4 May 2014; Published 2 June 2014

Academic Editor: Gerhard Litscher

Copyright (C) 2014 Mei-Ling Yeh et al. This is an open access article distributed under the Creative Commons Attribution License, which permits unrestricted use, distribution, and reproduction in any medium, provided the original work is properly cited.

\begin{abstract}
The earlier one starts to smoke, the more likely it is that one's tobacco use will increase. Either auricular acupressure or multimedia education could improve physiological health status and reduce smoking for young smokers. This study aimed to evaluate the effects of a 10-week auricular acupressure (AA) and interactive multimedia (IM) on smoking cessation in college smokers. A preand posttest control research design with two experiments (AA and IM) and one control was used. Thirty-two participants were in each of three groups. A significant difference from pretest to posttest among three groups was exhibited on carbon monoxide (CO), cotinine, and nicotine dependence. Scheffe's post hoc test found significances on CO in the AA between the IM and the control and cotinine and nicotine dependence between the AA and the control. After controlling the covariates, the main effect of the group was no difference in all outcomes. The interventions, especially AA, may contribute to a decrease of CO, cotinine, and nicotine dependence along with the time change. An analysis without controlling influences may overestimate interventional effects.
\end{abstract}

\section{Introduction}

Young adulthood is a critical period during which nicotine dependence and smoking behavior develop. About $21.5 \%$ of young adults become daily smokers [1]. Failure to stop smoking may result in increased smoking intensity and cumulative cigarette consumption [2], increased susceptibility to autoimmune diseases [3], higher risk of metabolic disorders [4], and early decline in lung function [2]. Forced expiratory volume in the first second (FEV1) is an early indicator of air flow limitation in asymptomatic smokers. Moderate to heavy smokers have a $15 \mathrm{ml} /$ year larger decline in FEV1 than in nonsmokers, and when decline was expressed as FEV1 percent predicted (FEV1\%), there is no gender difference [5]. FEV1 can recover after smoking cessation [2]. Previous studies, in addition to suggesting the measure of pulmonary function [6], found that carbon monoxide (CO), cotinine, and nicotine dependence would be normalized $[7,8]$.
According to the transtheoretical model of health behavior change [9], people in any stage of precontemplation, contemplation, preparation, and action maintain present readiness for changing smoking motivation and behavior. Smoking cessation intervention would help move upward the smokers' stages of change that were positively influenced by one's self-efficacy $[10,11]$. Moreover several factors influence the decision of young smokers to quit, for instance, years of smoking $[6,8]$, peer smoking $[7,12]$, and parental smoking [13-15]. The earlier one starts to smoke, the more likely it is that one's tobacco use will increase.

Auricular acupressure (AA) is a simple, noninvasive intervention that can decrease nicotine dependence and improve the refusal smoking self-efficacy $[13,16]$. Acupoint stimulation can increase endorphin [17] and serotonin [18] that are transmitted to the brain and particular organs in the rest of the body through nerves and meridian so as to modulate physiological reactions [19]. AA had positive effects 
on smoking cessation as measured by CO or cotinine $[8,20]$ but not in the others [21]. The systematic review concluded that no particular acupuncture is superior to any other [22]. However, the poor sensitivity of CO and cotinine levels as indicators of smoking activity has been reported in young smokers [23]. Outcome measures of AA in young smokers need further studies.

The flexible, convenient, and interesting content of interactive multimedia (IM) may help smokers accelerate the process of behavior change [24], improving positive cognition and attitude of smoking cessation [7], reinforcing selfefficacy to resist smoking [7], and contributing to a decreased nicotine dependence [25]. However, the effectiveness of IM in cessation remains controversial because of measurement issues, such as lack of biochemical verification [26].

\section{Purpose}

This study aimed to evaluate the main effects of AA and IM on smoking cessation in college smokers. Significant differences in FEV1\%, exhaled CO, serum cotinine, and nicotine dependence were hypothesized among the groups.

\section{Methods}

3.1. Research Design and Participants. This study used a preand posttest control research design with two experimental groups (one given AA and the other IM for 10 weeks) and one control group (receiving no intervention for 10 weeks). Participants were recruited on a voluntary basis from one university in the north of Taiwan and were nonrandomly assigned to one of three groups based on their preference. Inclusion criteria were enrolled in the study university: over 18 years, $\mathrm{CO}>6 \mathrm{ppm}$, serum cotinine $>100 \mathrm{ng} / \mathrm{mL}$, no abnormally shaped cutaneous lesions at the application sites, and not treated for smoking. Based on FEV1\% with a medium effect size $(f=0.3)$ and $80 \%$ power at $5 \%$ level of significance using repeated-measure 3 times, a total of 75 subjects would be minimally required. With an estimated loss of $20 \%, 90$ subjects were necessary.

3.2. Interventions. AA is a smoking cessation method involving pressure applied to six common auricular acupoints of shenmen, lung, stomach, mouth, subcortex, and hunger [6$8,13]$. The stimulation of the shenmen can raise endorphin and thus decrease withdrawal syndromes [19] and nicotine dependence [27]. Stimulation of the lung, stomach, mouth, and subcortex can normalize qi energy flow and harmonize the stomach and heart [28]. Stimulation of the hunger can prolong gastric motility and decrease appetite [19]. A seedembedding method was used to prolong stimulation at the selected acupoints. An adhesive patch containing the seed was replaced weekly. Participants were instructed to press each auricular acupoint for at least 1 minute per time and 3-5 times per day for 10 weeks. AA was given by a researcher who had earned 40 traditional Chinese medicine related formal credits and was validated by two physicians who practiced traditional Chinese medicine.
The format of IM contained files of text, animation, graphics, picture, sound, and image to provide instructions. The major contents of the program CD included the impact of smoking on health and the environment, information about smoking hazards, strategies for smoking cessation, and problems arising during the smoking cessation period and their solutions [7, 13]. The participants in the computer lab were given the instruction through IM for at least $20 \mathrm{~min}$ per time and once a week for 10 weeks. Instead of playing the 20 min whole video, the version of IM is capable of playing only the selected segments, which is designed to facilitate practice and review. Therefore, the participants could watch it without time limits and effectively select the part as needed.

3.3. Ethical Considerations. The ethical approval was obtained from the study university. Subjects who met the inclusion criteria were invited to participate. Informed consent was then obtained from all participants and assurance was given to them that the data would be handled anonymously. After completing the pretest of outcome measures, on the schedule the intervention was given. Participants were also provided verbal information concerning care of the ear seed. A posttest was conducted at the end of the intervention.

3.4. Outcome Measures. FEV1 is the amount of air which can be forcibly exhaled from the lungs in the first second of a forced expiratory maneuver [5]. Forced vital capacity (FVC) is the amount of air which can be forcibly and maximally exhaled out of the lungs after a maximal inhalation. FEV1\% is the ratio of FEV1 to FVC, with $75-80 \%$ in healthy adults. FEV1 and FVC were assessed lung function using a SpirolabII spirometer (Medical International Research, Rome, Italy). Exhaled $\mathrm{CO}$ and serum cotinine are objective biomarkers to distinguish smokers from nonsmokers [29]. Exhaled CO was measured by a Micro Medical meter (Micro Co., Rochester, Kent, England), with a cutoff value of under $6 \mathrm{ppm}$ used to identify nonsmokers [30]. Cotinine was determined by enzyme-linked immunosorbent assay (ELISA), the cotinine direct ELISA kit (Immunalysis Co., Pomona, CA), and an ELISA reader (Molecular Devices Co., Menlo Park, CA). Cotinine under $100 \mathrm{ng} / \mathrm{mL}$ indicated the absence of tobacco use [7].

The demographic characteristics involved age, age of initial smoking, age of regular smoking, times tried to quit smoking, peer smoking, family smoking, second-hand smoking, and parental education levels. Severity of nicotine dependence was measured using the FTND that has satisfactory validity and reliability [31]. The higher likelihood of nicotine dependence was indicated by higher score. A modified refusal smoking self-efficacy scale was used to measure the resistance to smoking in different circumstances [32] and has satisfactory validity and reliability [7]. A higher score indicated a greater likelihood that the individual could control smoking intention and behavior.

3.5. Data Analysis. Statistical analyses were performed using the IBM SPSS 20.0. The 5\% level of significance was used to confirm. Demographic data was analyzed by the descriptive 
TABLE 1: Demographic characteristics of the three groups.

\begin{tabular}{|c|c|c|c|c|}
\hline Variables/groups & $\begin{array}{c}\mathrm{AA} \\
\mathrm{M} \pm \mathrm{SD}, n(\%)\end{array}$ & $\begin{array}{c}\mathrm{IM} \\
\mathrm{M} \pm \mathrm{SD}, n(\%)\end{array}$ & $\begin{array}{c}\text { Control } \\
\mathrm{M} \pm \mathrm{SD}, n(\%)\end{array}$ & $P$ \\
\hline Age at study time (yr) & $23.47 \pm 3.07$ & $23.09 \pm 3.12$ & $22.72 \pm 3.01$ & 0.62 \\
\hline Age of initial smoking (yr) & $15.75 \pm 1.57$ & $15.53 \pm 1.14$ & $15.13 \pm 1.54$ & 0.21 \\
\hline Age of regular tobacco use (yr) & $16.56 \pm 1.63$ & $15.94 \pm 1.05$ & $16.03 \pm 1.20$ & 0.13 \\
\hline Times tried to quit smoking & $2.16 \pm 2.01$ & $1.44 \pm 1.85$ & $1.84 \pm 2.36$ & 0.40 \\
\hline Quitting smoking over $24 \mathrm{hr}$ & & & & 0.45 \\
\hline Yes & $25(78.1)$ & $21(65.6)$ & $21(65.6)$ & \\
\hline No & $7(21.9)$ & $11(34.4)$ & $11(34.4)$ & \\
\hline Peer smoking & & & & 0.50 \\
\hline Yes & $22(68.8)$ & $26(81.2)$ & $23(71.9)$ & \\
\hline No & $10(31.2)$ & $6(18.8)$ & $9(28.1)$ & \\
\hline Family smoking & & & & 0.84 \\
\hline Yes & $19(59.4)$ & $21(65.6)$ & $21(65.6)$ & \\
\hline No & $13(40.6)$ & $11(34.4)$ & $11(34.4)$ & \\
\hline With second-hand smoking & & & & 0.72 \\
\hline Yes & $22(68.8)$ & $20(62.5)$ & $23(71.9)$ & \\
\hline No & $10(31.2)$ & $12(37.5)$ & $9(28.1)$ & \\
\hline Father's education level & & & & 0.68 \\
\hline Junior high & $3(9.4)$ & $1(3.1)$ & $3(9.4)$ & \\
\hline Senior high & $11(34.4)$ & $10(31.3)$ & $12(37.5)$ & \\
\hline Vocational college & $12(37.5)$ & $16(50)$ & $11(34.4)$ & \\
\hline University and over & $6(18.8)$ & $5(15.6)$ & $6(18.7)$ & \\
\hline
\end{tabular}

$n=32$ in each group; AA: auricular acupressure; IM: interactive multimedia.

and univariate analysis. The $\chi^{2}$ tests and one-way ANOVA, when applicable, were conducted separately to compare the among-group difference in main outcome variables before (pretest) and after (posttest) the course of the intervention. A general linear mixed model was used fixed effects of intervention, time, and the interaction of intervention and time, and a random subject effect, followed by a Bonferroni test to compare the effects of smoking cessation.

\section{Results}

Of the 112 male participants recruited, 16 withdrew from the study for academic reasons, with the attrition rate of $14.3 \%$. Finally, 96 were in this study, with the mean age of 22.72-23.47 years at the start of the study, 15.13-15.75 years at smoking initiation, and 15.94 16.56 years when smoking became habitual. Of which, $69.79 \%$ participants quit smoking for more than $24 \mathrm{hr}$, with $1.81 \pm 0.22$ times tried to quit smoking. Table 1 shows the demographic homogeneity of the three groups.

Table 2 presents the results of FEV1\%, CO, cotinine, and nicotine dependence. Before the interventions, the mean FEV1\% was $89.66 \%$, with 15 participants having FEV1\% of less than $80 \%$. The results of ANOVA showed no significant difference among three groups at pretest on FEV1\% $(F=0.40$, $P=0.67), \mathrm{CO}(F=0.40, P=0.67)$, cotinine $(F=2.22$, $P=0.11)$, and nicotine dependence $(F=1.74, P=0.24)$. A significant difference from pretest to posttest among three groups was exhibited on $\mathrm{CO}(F=5.41, P=0.01)$, cotinine
$(F=4.39, P=0.02)$, and nicotine dependence $(F=10.50$, $P<0.001)$ but not in FEV1\% $(F=0.38, P=0.69)$. The Scheffe's post hoc test found significances on CO in the AA between the IM $(P=0.03)$ and the control $(P=0.02)$, on cotinine between the AA and the control $(P=0.02)$, and nicotine dependence between the AA and the control $(P<$ 0.01).

Table 3 presents the results of mixed models of FEV1\%, carbon monoxide, cotinine, and nicotine dependence. After controlling the variables of time, father smoking, peer smoking, refusal smoking self-efficacy, and/or smoking years, the result of the general linear mixed model on the main effect of group was not significant difference across time in FEV1\%, CO, cotinine, and nicotine dependence $(P>0.05)$. Regardless of interventions, the statistical significance in the main effect of time was significantly different in $\mathrm{CO}$ and nicotine dependence $(P<0.001)$. The interaction between group and time was significant in $\mathrm{CO}(P=0.02)$ and nicotine dependence $(P<0.001)$. The participants had no adverse effect associated with AA during this study period.

\section{Discussion}

5.1. Effects of the Interventions. This study found the effect of the AA and IM interventions on smoking cessation for college smokers in $\mathrm{CO}$, cotinine, and nicotine dependence, but not in FEV1\%. This was similar to other studies [8, 21]. However, the interventions reduced smoking behavior but did not lead to normalization of pulmonary function, and 
TABLE 2: FEV1\%, carbon monoxide, cotinine, and nicotine dependence at pre- and posttests.

\begin{tabular}{|c|c|c|c|}
\hline Variables & $\begin{array}{c}\mathrm{AA} \\
\mathrm{M} \pm \mathrm{SD}\end{array}$ & $\begin{array}{c}\mathrm{IM} \\
\mathrm{M} \pm \mathrm{SD}\end{array}$ & $\begin{array}{l}\text { Control } \\
\mathrm{M} \pm \mathrm{SD}\end{array}$ \\
\hline \multicolumn{4}{|l|}{ FEV1 \% } \\
\hline Pretest & $1.04 \pm 0.09$ & $1.00 \pm 0.10$ & $1.00 \pm 0.10$ \\
\hline Posttest & $1.04 \pm 0.09$ & $1.01 \pm 0.09$ & $1.02 \pm 0.09$ \\
\hline \multicolumn{4}{|c|}{ Carbon monoxide (ppm) } \\
\hline Pretest & $13.16 \pm 5.07$ & $12.81 \pm 4.13$ & $12.03 \pm 4.00$ \\
\hline Posttest & $10.31 \pm 4.28$ & $11.75 \pm 3.55$ & $11.09 \pm 3.64$ \\
\hline \multicolumn{4}{|c|}{ Cotinine (ng/mL) } \\
\hline Pretest & $732.44 \pm 814.11$ & $946.25 \pm 1074.39$ & $755.91 \pm 831.26$ \\
\hline Posttest & $407.69 \pm 496.68$ & $683.19 \pm 775.90$ & $756.06 \pm 807.32$ \\
\hline \multicolumn{4}{|c|}{ Nicotine dependence } \\
\hline Pretest & $5.09 \pm 2.19$ & $5.59 \pm 1.76$ & $6.16 \pm 1.71$ \\
\hline Posttest & $2.53 \pm 2.36$ & $4.09 \pm 1.89$ & $5.75 \pm 1.83$ \\
\hline
\end{tabular}

$n=32$ in each group; AA: auricular acupressure; IM: interactive multimedia; FEV1: forced expiratory volume in the first second.

TABLE 3: The results of mixed models of FEV1\%, carbon monoxide, cotinine, and nicotine dependence.

\begin{tabular}{lcccccccccccc}
\hline \multirow{2}{*}{ Parameters } & \multicolumn{3}{c}{ FEV1\% } & \multicolumn{4}{c}{ Carbon monoxide } & \multicolumn{3}{c}{ Cotinine } & \multicolumn{3}{c}{ Nicotine dependence } \\
& Beta & SE & $F$ & Beta & SE & $F$ & Beta & SE & $F$ & Beta & SE & $F$ \\
\hline Intercept & 1.03 & 0.02 & $199.49^{* * *}$ & 7.78 & 1.35 & $47.87^{* * *}$ & -84.79 & 264.26 & 0.02 & 32.00 & 3.51 & $157.11^{* * *}$ \\
Time & -0.02 & 0.02 & 1.36 & 0.90 & 0.46 & $20.66^{* * *}$ & -36.58 & 80.17 & 1.22 & -1.60 & 1.03 & $41.11^{* * *}$ \\
Father smoking & -0.07 & 0.03 & $6.57^{*}$ & -1.63 & 1.33 & 1.51 & 129.26 & 265.89 & 0.24 & 0.90 & 3.55 & 0.06 \\
Peer smoking & & & & 0.41 & 0.90 & 0.21 & 47.81 & 180.98 & 0.07 & 4.74 & 2.42 & $3.83^{*}$ \\
Self-efficacy & & & & 0.10 & 0.12 & 0.67 & 89.67 & 21.85 & $16.84^{* * *}$ & -0.45 & 0.28 & 2.49 \\
Smoking years & & & & 0.42 & 0.13 & $11.25^{* *}$ & 44.21 & 25.24 & 3.07 & -0.24 & 0.34 & 0.52 \\
\hline
\end{tabular}

FEV1: forced expiratory volume in the first second; ${ }^{*} P<0.05,{ }^{* *} P<0.01,{ }^{* * *} P<0.001$.

this effect differed from one of the other studies [5]. FEV1 should peak in early adulthood; however, this study found a preintervention decline in pulmonary function in the sample of young college smokers. This result is in agreement with other studies [33]. With age, the decline is even more obvious [34].

The interventions, especially AA, could assist college smokers to decrease their $\mathrm{CO}$, cotinine, and nicotine dependence. It seems AA may facilitate progression to more advanced stages of changing smoking behavior. The effect of IM on smoking cessation is slightly supported in this study, which is not entirely in agreement with other studies $[8,35,36]$. Maybe this is because previous studies lacked biochemical verification [26]. In addition, lack of effects may be due to adherence and frequency of use by participants, especially male participants [37]. Therefore, this study suggests that frequency of use and process may be factors influencing the efficacy of intervention. In addition, using IM approaches alone for smoking cessation may not be effective. Some studies support the use of combined rather than single interventions to stop smoking $[7,8,38]$. It is noted that the effect of AA on smoking cessation became not obvious after controlling the variables of time, father smoking, peer smoking, refusal smoking self-efficacy, and/or smoking years in the statistical analysis in this study. As mentioned previously those confounders and the time effect should be considered [6-15]. This result may exhibit the correction for possible overestimation of the effect of AA and IM.

5.2. Measurement Issues of Smoking Cessation. Some issues related to the assay of these biomarkers in young smokers have surfaced. First, the specificity of CO and cotinine was high, but their sensitivity was low in this study. The levels of $\mathrm{CO}$ and cotinine were below the nonsmoking cutoff value in 6 and 11 participants, respectively, and were both below their cutoff values in 2 participants. This finding is similar to that found in one previous study [29]. The biological halflife of CO is $4-5 \mathrm{hr}$ in sedentary adults. Physical activity and rate of pulmonary ventilation affect $\mathrm{CO}$ elimination. The half-life could be as brief as $1 \mathrm{hr}$ during exercise [39]. CO might also become a less sensitive biomarker if smoking behavior was restricted in a nonsmoking environment. In addition, exposure to traffic exhaust and other pollutants resulted in exhaled CO levels of 2-6 ppm [35]. Cotinine has a long biological half-life (15-40 hr) and it would increase and persist when smoking becomes a regular habit [29], which may contribute to a decrease in its sensitivity as a biomarker of change in smoking activity. Second, this study and another study [30] found that $\mathrm{CO}$ and cotinine were less correlated although both were sensitive indicators of 
nicotine dependence [31]. Although these two may be suitable as biochemical markers, other indicators are required.

5.3. Study Limitations. The potential threats to validity included selection and treatment assignment bias. The Hawthorne effect was also a threat because participants were aware that they were being observed. Thus, findings should be interpreted with caution because of the limitations of the study design. This study had the effect of smoking cessation during a short period, but a long-standing effect remains unknown. Longitudinal studies in young smokers of various ages, in different genders and in various geographic locales, are recommended. Although this study supports the effect of smoking cessation, this effect was not obvious after adjusting for the covariates. Future studies should clarify the influences of these covariates. Additional studies are needed to further validate the impact of interventions on smoking cessation.

\section{Conclusion}

A review of the literature indicates that this study is the first to examine the effects and the measures of AA and IM on FEV1, CO, cotinine, and nicotine dependence as biomarkers of smoking cessation in college smokers. The interventions, especially AA, may contribute to a decrease of CO, cotinine, and nicotine dependence along with the time change. However, the refusal smoking self-efficacy and smoking years of college smokers, as well as the smoking habits of their fathers and peers are important influences on smoking cessation. An analysis without controlling influences may overestimate interventional effects. Appropriate, even combined, smoking cessation interventions should be considered. Some issues related to the assay of biomarkers have surfaced.

\section{Conflict of Interests}

The authors declare that there is no conflict of interests regarding the publication of this paper.

\section{Authors' Contribution}

Jaung-Geng Lin and Mei-Ling Chung equally contributed to this work as cocorrespondent authors.

\section{References}

[1] D. Hammond, "Smoking behaviour among young adults: beyond youth prevention," Tobacco Control, vol. 14, no. 3, pp. 181-185, 2005.

[2] S. Chinn, D. Jarvis, R. Melotti et al., "Smoking cessation, lung function, and weight gain: a follow-up study," The Lancet, vol. 365, no. 9471, pp. 1629-1635, 2005.

[3] C. A. Whetzel, E. J. Corwin, and L. C. Klein, "Disruption in Th1/Th2 immune response in young adult smokers," Addictive Behaviors, vol. 32, no. 1, pp. 1-8, 2007.

[4] C. M. Kung, H. L. Wang, and Z. I. Tseng, "Cigarette smoking exacerbates health problems in young men," Clinical and Investigative Medicine, vol. 31, no. 3, pp. E138-E149, 2008.
[5] N. R. Anthonisen, J. E. Connett, and R. P. Murray, "Smoking and lung function of Lung Health Study participants after 11 years," American Journal of Respiratory and Critical Care Medicine, vol. 166, no. 5, pp. 675-679, 2002.

[6] M. L. Yeh, C. Y. Chang, N. F. Chu, and H. H. Chen, "A sixweek acupoint stimulation intervention for quitting smoking," American Journal of Chinese Medicine, vol. 37, no. 5, pp. 829836, 2009.

[7] H. H. Chen, M. L. Yeh, and Y. H. Chao, "Comparing effects of auricular acupressure with and without an Internet-assisted program on smoking cessation and self-efficacy of adolescents," Journal of Alternative and Complementary Medicine, vol. 12, no. 2, pp. 147-152, 2006.

[8] Y. Z. Wang, H. H. Chen, M. L. Yeh, and S. D. Lin, "Auricular acupressure combined with multimedia instruction or alone for quitting smoking in young adults: a quasi-experimental study," International Journal of Nursing Studies, vol. 47, no. 9, pp. 10891095, 2010.

[9] J. O. Prochaska, C. C. DiClemente, and J. C. Norcross, "In search of how people change: applications to addictive behaviors," American Psychologist, vol. 47, no. 9, pp. 1102-1114, 1992.

[10] S. Andersen, "Adding addiction to the transtheoretical model for smoking cessation," Addictive Behaviors, vol. 32, no. 5, pp. 1099-1104, 2007.

[11] E. L. O’Hea, E. D. Boudreaux, S. K. Jeffries, C. L. Carmack Taylor, I. C. Scarinci, and P. J. Brantley, "Stage of change movement across three health behaviors: the role of selfefficacy," American Journal of Health Promotion, vol. 19, no. 2, pp. 94-102, 2004.

[12] J. S. Tucker, P. L. Ellickson, M. Orlando, and D. J. Klein, "Predictors of attempted quitting and cessation among young adult smokers," Preventive Medicine, vol. 41, no. 2, pp. 554-561, 2005.

[13] H. H. Chen and M. L. Yeh, "Developing and evaluating a smoking cessation program combined with an Internet-assisted instruction program for adolescents with smoking," Patient Education and Counseling, vol. 61, no. 3, pp. 411-418, 2006.

[14] M. C. Hu, M. Davies, and D. B. Kandel, "Epidemiology and correlates of daily smoking and nicotine dependence among young adults in the United States," American Journal of Public Health, vol. 96, no. 2, pp. 299-308, 2006.

[15] J. T. Macy, D. Seo, L. Chassin, C. C. Presson, and S. J. Sherman, "Prospective predictors of long-term abstinence versus relapse among smokers who quit as young adults," American Journal of Public Health, vol. 97, no. 8, pp. 1470-1475, 2007.

[16] C. Y. Chang, N. F. Chu, and M. L. Yeh, "Acupoint stimulation as a smoking cessation therapy," Journal of Nursing, vol. 53, no. 5, pp. 63-68, 2006.

[17] B. Pan, J. M. Castro-Lopes, and A. Coimbra, "Activation of anterior lobe corticotrophs by electroacupuncture or noxious stimulation in the Anaesthetized rat, as shown by colocalization of Fos protein with ACTH and $\beta$-endorphin and increased hormone release," Brain Research Bulletin, vol. 40, no. 3, pp. 175182, 1996.

[18] K. Yoshimoto, F. Fukuda, M. Hori et al., "Acupuncture stimulates the release of serotonin, but not dopamine, in the rat nucleus accumbens," Tohoku Journal of Experimental Medicine, vol. 208, no. 4, pp. 321-326, 2006.

[19] M. L. Yeh, H. H. Chen, and I. H. Lin, Contemporary Meridians and Acupoints in Practice, Farseeing, Taipei, Taiwan, 2004. 
[20] C. Yiming, Z. Changxin, W. S. Ung, Z. Lei, and L. S. Kcan, "Laser acupuncture for adolescent smokers-a randomized doubleblind controlled trial," American Journal of Chinese Medicine, vol. 28, no. 3-4, pp. 443-449, 2000.

[21] D. He, J. I. Medbo, and A. T. Hostmark, "Effect of acupuncture on smoking cessation or reduction: an 8-month and 5-year follow-up study," Preventive Medicine, vol. 33, no. 5, pp. 364372, 2001.

[22] A. R. White, H. Rampes, and J. L. Campbell, "Acupuncture and related interventions for smoking cessation," Cochrane Database of Systematic Reviews, no. 1, Article ID CD000009, 2006.

[23] H. A. Lando, P. G. McGovern, S. H. Kelder, R. W. Jeffery, and J. L. Forster, "Use of carbon monoxide breath validation in assessing exposure to cigarette smoke in a worksite population," Health Psychology, vol. 10, no. 4, pp. 296-301, 1991.

[24] M. L. Yeh, H. H. Chen, and H. F. Chang, "The effect of internet assisted smoking cessation program among adolescents," Formosan Journal of Medicine, vol. 6, no. 5, pp. 648-660, 2002.

[25] D. J. Fritz, S. B. Hardin, P. A. Gore, and D. Bram Jr., "A computerized smoking cessation intervention for high school smokers," Pediatric Nursing, vol. 34, no. 1, pp. 13-17, 2008.

[26] H. Brendryen and P. Kraft, "Happy Ending: a randomized controlled trial of a digital multi-media smoking cessation intervention," Addiction, vol. 103, no. 3, pp. 478-484, 2008.

[27] L. Hsu and T. Yang, "Auricular acupressure for 257 cases in smoking cessation,” Shanghai Acupunct Journal, vol. 19, pp. 2324, 2000.

[28] H. M. Chen and X. L. Gao, The Acupuncture Point on the Ear Treats All the Various Illnesses, Big-Exhibition Press, Taipei, Taiwan, 2005.

[29] K. R. Stevens and L. R. Muñoz, "Focus on research methodscigarette smoking: evidence to guide measurement," Research in Nursing and Health, vol. 27, no. 4, pp. 281-292, 2004.

[30] R. H. Secker-Walker, P. M. Vacek, B. S. Flynn, and P. B. Mead, "Exhaled carbon monoxide and urinary cotinine as measures of smoking in pregnancy," Addictive Behaviors, vol. 22, no. 5, pp. 671-684, 1997.

[31] C. L. Huang, H. H. Lin, and H. H. Wang, "Evaluating screening performances of the Fagerstrom tolerance questionnaire, the Fagerstrom test for nicotine dependence and the heavy smoking index among Taiwanese male smokers," Journal of Clinical Nursing, vol. 17, no. 7, pp. 884-890, 2008.

[32] Q. Y. Liao, The tobacco harms the synthesis questionnaire to edit the research [M.S. thesis], National Taiwan University, Taipei, Taiwan, 1994.

[33] G. Parkes, T. Greenhalgh, M. Griffin, and R. Dent, "Effect on smoking quit rate of telling patients their lung age: the Step2quit randomised controlled trial," BMJ, vol. 336, no. 7644, pp. 598$600,2008$.

[34] H. Omori and Y. Morimoto, "Effects of smoking habits on pulmonary function. Cross-sectional and longitudinal studies in male subjects on medical check-up," The journal of the Japanese Respiratory Society, vol. 42, no. 4, pp. 306-312, 2004.

[35] N. L. Benowitz, P. Jacob, K. Ahijevych et al., "Biochemical verification of tobacco use and cessation," Nicotine and Tobacco Research, vol. 4, no. 2, pp. 149-159, 2002.

[36] J. F. Etter, "Comparing the efficacy of two internet-based, computer-tailored smoking cessation programs: a randomized trial," Journal of Medical Internet Research, vol. 7, no. 1, p. e1, 2005.
[37] C. A. Patten, E. Rock, T. M. Meis et al., "Frequency and type of use of a home-based, internet intervention for adolescent smoking cessation," Journal of Adolescent Health, vol. 41, no. 5, pp. 437-443, 2007.

[38] I. D. Bier, J. Wilson, P. Studt, and M. Shakleton, "Auricular acupuncture, education, and smoking cessation: a randomized, sham-controlled trial," American Journal of Public Health, vol. 92, no. 10, pp. 1642-1647, 2002.

[39] S. E. Marcus, K. Pahl, Y. Ning, and J. S. Brook, "Pathways to smoking cessation among African American and Puerto Rican young adults," American Journal of Public Health, vol. 97, no. 8, pp. 1444-1448, 2007. 


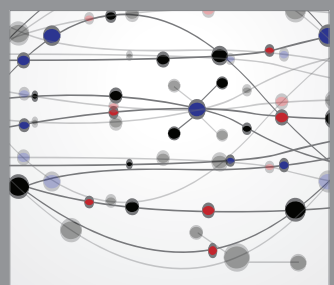

The Scientific World Journal
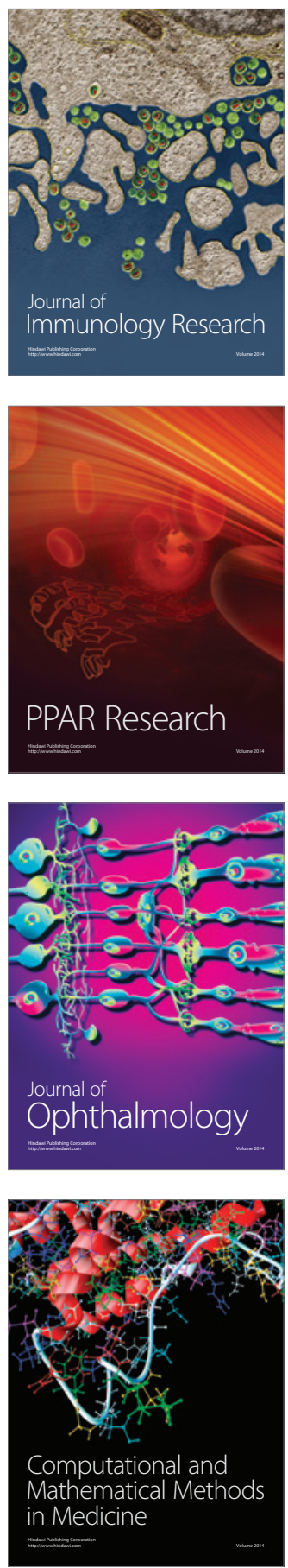

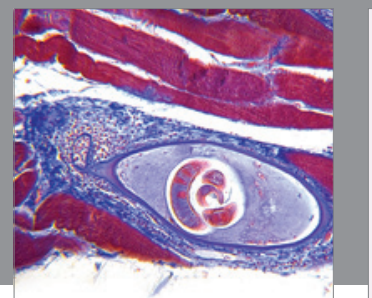

Gastroenterology

Research and Practice
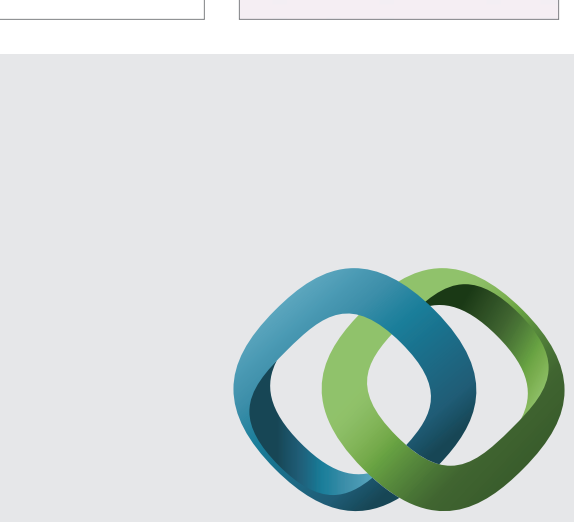

\section{Hindawi}

Submit your manuscripts at

http://www.hindawi.com
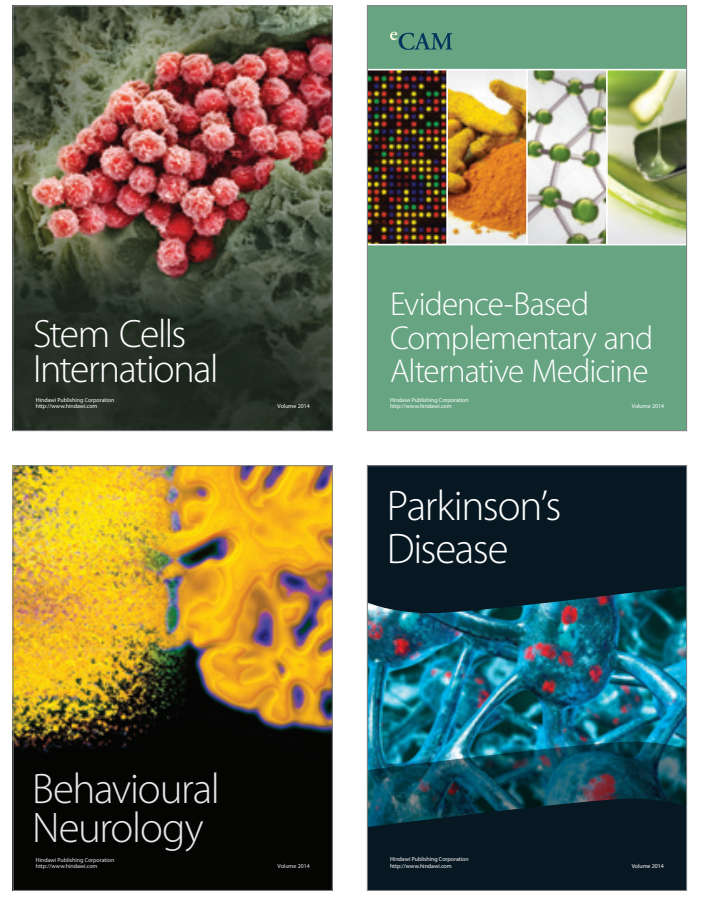
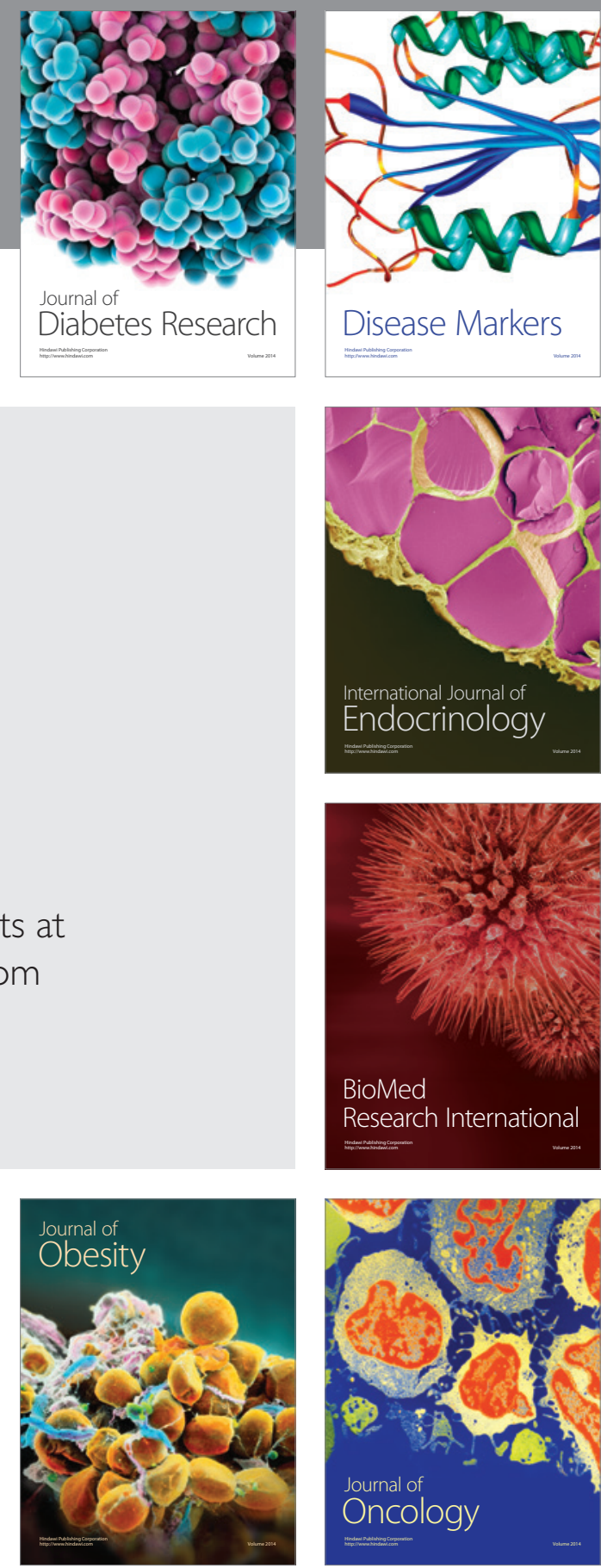

Disease Markers
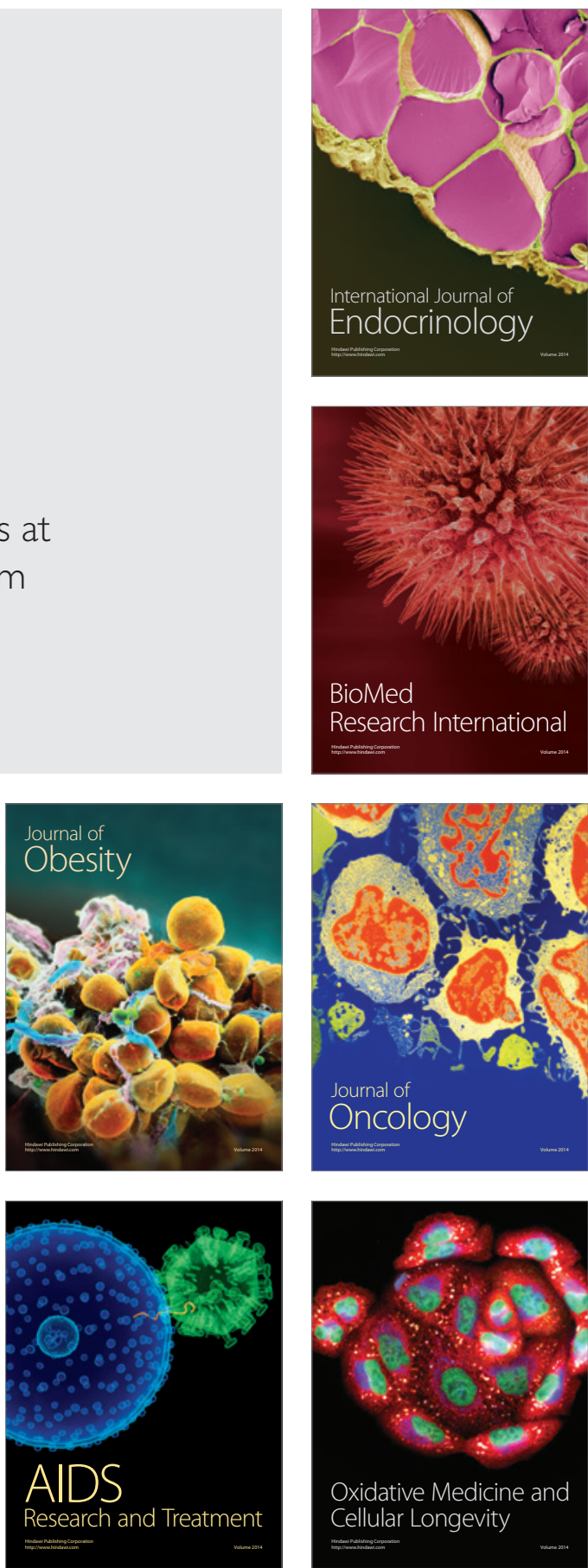